La Revue

des Droits

de l'Homme

\section{La Revue des droits de l'homme}

Revue du Centre de recherches et d'études sur les droits fondamentaux

Actualités Droits-Libertés | 2014

\title{
De nouvelles menaces sur le processus de renforcement du système interaméricain des droits de l'homme
}

Cour interaméricaine des droits de l'homme (CIADH)

Juana María Ibáñez Rivas

\section{OpenEdition}

Journals

Édition électronique

URL : http://journals.openedition.org/revdh/977

DOI : $10.4000 /$ revdh. 977

ISSN : 2264-119X

Éditeur

Centre de recherches et d'études sur les droits fondamentaux

Référence électronique

Juana María Ibáñez Rivas, « De nouvelles menaces sur le processus de renforcement du système interaméricain des droits de l'homme », La Revue des droits de l'homme [En ligne], Actualités DroitsLibertés, mis en ligne le 21 novembre 2014, consulté le 20 avril 2019. URL : http:// journals.openedition.org/revdh/977 ; DOI : 10.4000/revdh.977

Ce document a été généré automatiquement le 20 avril 2019.

Tous droits réservés 


\title{
De nouvelles menaces sur le processus de renforcement du système interaméricain des droits de l'homme
}

Cour interaméricaine des droits de l'homme (CIADH)

\author{
Juana María Ibáñez Rivas
}

1 Conformément à l'article 62.1 de la Convention américaine des droits de l'homme, «tout Etat partie peut, au moment du dépôt de son instrument de ratification ou d'adhésion à la [CADH], ou à tout autre moment ultérieur, déclarer qu'il reconnaît comme obligatoire, de plein droit et sans convention spéciale, la compétence de la [Cour interaméricaine des droits de l'homme] pour connaître de toutes les espèces relatives à l'interprétation ou à l'application de la [dite CADH] ». La République Dominicaine a respectivement signé et ratifié la Convention américaine des droits de l'homme les 7 septembre et 25 décembre 1977. Mais ce n'est que le 19 février 1999 que le Président de la République de l'époque a accepté, au nom de l'Etat, de reconnaître " comme obligatoire de plein droit et sans convention spéciale », la compétence de la CIADH.

Cependant, le 25 novembre 2005, un groupe de citoyens a initié une action directe d'inconstitutionnalité concernant l'instrument d'acceptation de la compétence contentieuse de la CIADH en alléguant, entre autres, que l'acte du Président de la République de l'époque n'avait pas été ratifié par le Congrès de la République en contravention des dispositions de la Constitution politique et de l'article 46 de la Convention de Vienne sur le droit des traités de 1969. Pour cette raison, parmi d'autres, l'instrument d'acceptation de la compétence contentieuse de la Cour interaméricaine des droits de l'homme serait nul et n'engagerait pas la responsabilité de l'Etat. Le 4 novembre 2014, le Tribunal constitutionnel a rendu dans sa décision sur l'action directe d'inconstitutionnalité en déclarant, par dix voix contre trois, que l'instrument d'acceptation était inconstitutionnel. La gravité de cette situation appelle une analyse des considérations qui ont amené le Tribunal constitutionnel à prendre une telle décision 
$\left(1^{\mathrm{o}}\right)$, tout en évaluant la portée de celle-ci concernant la participation de la République Dominicaine au système interaméricain des droits de l'homme $\left(2^{\circ}\right)$.

\section{$1 \%$ - La décision du Tribunal constitutionnel de la République Dominicaine mise en perspective : Plus que des arguments juridiques?}

Bien que l'action directe d'inconstitutionnalité contre l'instrument d'acceptation de la compétence contentieuse de la Cour interaméricaine des droits de l'homme ait été introduite le 25 novembre 2005, la décision a été rendue presque neuf ans plus tard, le 4 novembre 2014. Mais cette décision du Tribunal constitutionnel a été rendue deux semaines seulement après la notification et la publication d'un nouvel arrêt de la CIADH qui condamne la République Dominicaine au sujet de situation des personnes haïtiennes et dominicaines d'ascendance haïtienne dans le pays. Par conséquent, outre les arguments juridiques propres à la décision (A), il est inévitable de prendre en compte les éventuels facteurs extrajudiciaires qui peuvent avoir joué un rôle dans la décision du Tribunal constitutionnel (B).

\section{A - Le contenu et la portée de la décision : Des justifications juridiques à la lumière du droit interne et du droit international public}

4 La décision de la majorité des juges du Tribunal constitutionnel déclarant l'inconstitutionnalité de l'instrument d'acceptation de la compétence contentieuse de la CIADH est fondée sur deux arguments principaux qui supposent une lecture conjointe du droit interne dominicain et de la Convention de Vienne. En premier lieu, la majorité a invoqué l'exception à l'interdiction pour un Etat de s'appuyer sur les règles du droit interne pour échapper à ses responsabilités en vertu des conventions internationales, contenue à l'article 46.1 de la Convention de Vienne. Ce texte souligne qu'une violation "manifeste» concernant une règle du droit interne "d'importance fondamentale " peut être invoquée comme viciant le consentement de l'Etat à être partie à un traité (\$9.5). Selon la majorité, les obligations qu'assument les Etats parties à la Convention américaine des droits de l'homme face à la Cour interaméricaine des droits de l'homme découlent de l'approbation et de la ratification d'un instrument international « additionnel » à cette Convention (§ 9.13). Pour la majorité, même si l'instrument d'acceptation de la compétence contentieuse de la CIADH «est un acte unilatéral nonautonome réalisé dans le cadre de la $\mathrm{CADH}$, celui-ci à la même force que les conventions internationales ». Par conséquent, conformément à ce qui est établi dans la Constitution politique, la volonté du pouvoir exécutif de conclure des traités requiert l'approbation du Congrès national. Selon l'arrêt du Tribunal constitutionnel, l'inconstitutionnalité de l'instrument d'acceptation provient du fait qu'en l'espèce, cette «vérification » n'a pas eu lieu (\$§9.18-9.19).

En second lieu, la majorité a rejeté une éventuelle violation des principes d'estoppel et de forum prorogatum en relation avec les actes et activités antérieurs de la République Dominicaine devant la CIADH. Selon le Tribunal constitutionnel, l'acceptation à de nombreuses reprises de la compétence des représentations de l'Etat 
dominicain devant la CIADH, la reconnaissance de cette compétence par la Cour suprême du pays, la proposition puis l'élection d'une juge dominicaine à un poste de magistrat de la $\mathrm{CIADH}^{1}$, ainsi que la reconnaissance du caractère contraignant des décisions de la CIADH par le Tribunal constitutionnel lui-même, se sont toutes fondées sur la présomption de légalité de l'instrument d'acceptation de la compétence contentieuse de la CIADH (§§9.8-9.9).

La surprenante décision de la majorité des juges du Tribunal constitutionnel est contrebalancée par les opinions dissidentes des trois juges qui ont voté contre la décision, Hermógenes Acosta de los Santos, Ana Isabel Bonilla Hernández, et Katia Miguelina Jiménez Martínez. De manière générale, les trois opinions dissidentes se rejoignent en considérant que la majorité a confondu un «traité international » avec un «acte unilatéral ». Ainsi, selon les juges dissidents, l'approbation du Congrès national n'était pas nécessaire en l'espèce car, selon l'article 62.1 de la CADH que l'Etat dominicain a accepté en la ratifiant en 1977, la République Dominicaine pouvait accepter la compétence contentieuse de la CIADH sans recourir à une convention spéciale ou aux mêmes formalités que celles requises pour un traité. De cette façon, pour les juges dissidents, l'instrument d'acceptation de la compétence contentieuse de la CIADH est un acte juridique non autonome qui trouve sa source dans la signature, et la ratification qui s'en est suivie par le Congrès national, de la Convention américaine des droits de l'homme. En tout état de cause, pour ces juges, l'instrument d'acceptation a été élaboré par le Président de la République, qui est investi des pouvoirs nécessaires pour signer de tels actes en vertu de la Constitution politique et de l'article 7 de la Convention de Vienne. En ce sens, les trois juges ont considéré que l'Etat ne pouvait alléguer la nullité de l'acte juridique unilatéral non autonome en question car ce serait une violation claire des principes d'estoppel et de forum prorogatum, l'Etat ayant prétendu pendant quinze ans que celui-ci était valide (Opinions dissidentes de Hermógenes Acosta $\$ \$ 17,20$, 22-29; Ana Isabel Bonilla §§2.6-2.7; et Katia Miguelina Jiménez Martínez \$§4.1.7, 4.1.9, 4.1.10, 4.1.14, 4.2.5, 4.2.8, 4.2.11, 4.4.6).

Il est important de noter qu'en plus d'avoir manifesté leur désaccord avec les considérations juridiques de la majorité, deux des juges dissidents ont mis en évidence une situation irrégulière quant à la publication de l'arrêt du Tribunal constitutionnel. En effet, contrairement aux dispositions de la Loi Organique du TC et des Procédures Constitutionnelles $^{2}$, et de la Constitution politique elle-même, la formation plénière du Tribunal constitutionnel a décidé, à la majorité, d'ordonner la publication de l'arrêt sans que deux des trois juges de la minorité aient eu l'occasion de rédiger leur opinion dissidente respective et, de plus, sans que la copie intégrale de l'arrêt leur soit dûment notifiée. Ces opinions ont été incluses postérieurement sur le site web du Tribunal constitutionnel. Ainsi, il est particulièrement curieux que le Tribunal constitutionnel ait déclaré que l'instrument d'acceptation de la compétence contentieuse de la CIADH était nul, mais que la décision d'inconstitutionnalité ait été publiée sans respecter les formalités exigées par le droit interne. Cette irrégularité renforce l'hypothèse qu'il existerait des motivations extrajudiciaires derrière la décision du Tribunal constitutionnel. 


\section{B - Les arrêts de la CIADH condamnant la République Dominicaine : La véritable motivation de la décision du Tribunal constitutionnel ?}

8 La CIADH a rendu quatre arrêts contre la République Dominicaine, dont trois se rapportent à la situation des personnes haïtiennes ou dominicaines d'ascendance haïtienne dans le pays: Mineures Yean et Bosico ${ }^{3}$, Nadege Dorzema et autres ${ }^{4}$ et, plus récemment, Personnes dominicaines et haïtiennes expulsées ${ }^{5}$. Ce dernier arrêt a été rendu le 24 août 2014, et notifié et publié le 22 octobre 2014, soit deux semaines avant la décision du Tribunal constitutionnel. Cette affaire concerne les privations de liberté illégales et arbitraires et les expulsions sommaires de la République Dominicaine à Haïti des personnes haïtiennes et dominicaines d'ascendance haïtienne, sans procès équitable et sans accès à un recours effectif pour garantir leurs droits, qui ont eu lieu entre 1999 et 2000. En ce sens, la CIADH a déterminé qu'à l'époque des faits de l'affaire, les personnes haïtiennes et d'ascendance haïtienne étaient souvent pauvres et sans papiers. De plus, elles souffraient fréquemment de traitements péjoratifs ou discriminatoires, y compris de la part des autorités, ce qui aggravait leur situation de vulnérabilité. En outre, la CIADH a conclu à l'existence, au moins durant une période d'environ une décennie à partir de 1990, d'un schéma systématique d'expulsions, y compris par des actes collectifs, d'haïtiens et de personnes d'ascendance haïtienne, reflétant une tendance discriminatoire dans le pays.

9 La CIADH a déterminé, entre autres, que la République Dominicaine avait manqué à son obligation d'adopter des mesures de droit interne (article 2 de la CADH) en relation à un arrêt du Tribunal constitutionnel rendu le 23 septembre $2013(\mathrm{TC} / 0168 / 13)^{6}$ et à certains articles de la Loi No. 169-14 du 23 mai 20147, adoptée sur la base dudit arrêt, qui considéraient comme «étrangers » les personnes nées en République Dominicaine dont les parents étaient des étrangers en situation migratoire irrégulière. Ces dispositions établissaient ainsi une situation de discrimination à la lumière de la $\mathrm{CADH}$ violant le droit à l'égalité devant la loi et d'autres droits reconnus dans la CADH. La CIADH a noté que l'irrégularité concernait les parents, et non les personnes nées en République Dominicaine, ce qui, en soi, n'était pas une justification valable pour distinguer entre les personnes nées sur le territoire dominicain dont les parents étaient des étrangers en situation migratoire régulière et celles dont les parents étaient en situation migratoire irrégulière. En ce sens, dans le cadre de la réparation intégrale, la CIADH a ordonné à l'Etat, entre autres, d'adopter les mesures de droit interne nécessaires pour éviter que l'arrêt du Tribunal constitutionnel du 23 septembre 2013 (TC/0168/13) et la Loi No. 169-14 continuent à produire des effets juridiques ; adopter les mesures nécessaires pour abroger toute disposition de toute nature, tant constitutionnelle, législative, réglementaire, administrative, ou toute pratique, ou décision, ou interprétation qui établisse ou qui ait pour effet que le séjour irrégulier des parents étrangers motive le refus de la nationalité dominicaine aux enfants nés sur le territoire de la République Dominicaine; et adopter des mesures législatives, y compris, si nécessaire, constitutionnelles, administratives et toutes autres mesures nécessaires, pour réglementer une procédure d'enregistrement de naissance accessible et simple, afin d'assurer que toutes les personnes nées en République Dominicaine puissent être enregistrées immédiatement après la naissance indépendamment de leur ascendance ou de l'origine et du statut d'immigration de leurs parents. 
10 La réaction de la République Dominicaine ne s'est pas faite attendre. Ainsi, le lendemain de la publication de l'arrêt, le 23 octobre 2014, le gouvernement dominicain a rejeté la décision de la CIADH.

11 La Commission interaméricaine a souligné que le rejet de l'Etat s'établissait dans un contexte de manquement par celui-ci aux décisions du système interaméricain en matière de violations des droits de l'homme résultant de la situation de discrimination structurelle contre les personnes haïtiennes et d'ascendance haïtienne vivant dans le pays $^{8}$. Ainsi, il n'est pas surprenant que l'opinion dissidente du juge Katia Miguelina Jiménez Martínez ait remarqué que la décision du Tribunal constitutionnel déclarant l'inconstitutionnalité de l'instrument d'acceptation de la compétence contentieuse de la CIADH a été rendue dans un contexte historique où le pays a été condamné par la CIADH, de sorte que, pour le juge Jiménez Martínez, la décision du Tribunal constitutionnel s'apparente " plus à un acte de réaction qu'à un raisonnement juridique $»(\$ 4.2 .12)$. Tribunal constitutionnel, son impact sur le système interaméricain est incontestable.

\section{$2 \%$ Les conséquences de la décision du Tribunal constitutionnel : Des effets notables envers la CIADH et au sein du système interaméricain}

La décision du Tribunal constitutionnel a pris soin de noter que la déclaration d'inconstitutionnalité de l'instrument d'acceptation de la compétence contentieuse de la CIADH «ne remet pas en question" l'adhésion et la ratification de la République Dominicaine à la $\mathrm{CADH}$, soulignant ainsi que le Tribunal constitutionnel «partage les postulats, les principes, les normes, les valeurs et les droits » de la CADH, lesquels «continueront à être normalement appliqués, respectés et pris en considération » par cette juridiction (\$9.21). Toutefois, la jurisprudence de la CIADH semblerait indiquer qu'il ne suffit pas d'une éventuelle déclaration d'inconstitutionnalité de l'instrument d'acceptation de sa compétence contentieuse pour éviter l'exercice de la même par rapport au pays concerné (A). En tout état de cause, indépendamment des conséquences par rapport à l'exercice de la compétence contentieuse de la CIADH, la décision du Tribunal constitutionnel a déjà des effets dans le processus de renforcement du système interaméricain (B).

\section{A - L'inconstitutionnalité de l'acceptation de la compétence contentieuse de la $\mathrm{CIADH}$ : Un processus incomplet selon la jurisprudence?}

14 La CIADH a déjà eu l'occasion de se prononcer sur des situations similaires dans lesquelles un État a tenté de se désengager de l'exercice de sa compétence contentieuse. Par 
exemple, par résolution législative du Congrès de la République du 8 juillet 1999, le Pérou a indiqué qu'il retirait sa déclaration d'acceptation de la compétence contentieuse de la CIADH avec "effet immédiat ». La CIADH s'est prononcée sur ce retrait à travers deux arrêts de compétence dans deux affaires contre le Pérou, Ivcher Bronstein ${ }^{9}$ et Tribunal constitutionnel ${ }^{10}$ du 24 septembre 1999, déclarant que la tentative de retrait du Pérou était irrecevable en vertu des dispositions de la CADH. En janvier 2001, avec le gouvernement de transition démocratique, le Pérou a annulé la déclaration contestée de 1999.

15 Ainsi, depuis 1999, la CIADH a établi que c'est elle seule qui peut se prononcer sur un " prétendu retrait » de la déclaration d'acceptation de sa compétence contentieuse et ses effets juridiques car, comme tout organe avec des compétences juridictionnelles, elle «a le pouvoir inhérent de déterminer la portée de sa propre compétence » (compétence de la compétence) ${ }^{11}$. Selon la CIADH, une objection ou tout autre acte pris par un État dans le but d'affecter sa compétence "est inoffensif " parce qu'en toutes circonstances, la CIADH détient la compétence de la compétence étant «maitresse de sa juridiction $»^{12}$. Par conséquent, pour la CIADH, l'acceptation de sa compétence contentieuse est immuable (correspondant, selon la CIADH, à une " cláusula pétrea »), et n'admet pas de limitations qui ne soient pas expressément énoncées à l'article 62.1 de la CADH ${ }^{13}$. De cette manière, à travers son interprétation de l'article 62.1 de la CADH, la CIADH a établi qu'aucune règle de la $\mathrm{CADH}$ n'habilite expressément les États partie à retirer leur déclaration d'acceptation de sa compétence contentieuse ${ }^{14}$.

16 En outre, la CIADH a indiqué que dans le fonctionnement du système de protection consacré dans la $\mathrm{CADH}$, la clause facultative d'acceptation de sa compétence contentieuse revêt une importance particulière, puisque l'État qui s'y soumet reste lié à l'intégrité de la $\mathrm{CADH}^{15}$. Selon la CIADH, il n'est pas possible de comparer un acte juridique unilatéral effectué dans le cadre des relations purement interétatiques qui se réalise par lui-même de manière autonome avec un acte juridique unilatéral effectué dans le cadre du droit conventionnel, comme l'acceptation d'une clause facultative de la compétence contentieuse d'un tribunal international, puisque cette acceptation est déterminée et conditionnée par le traité lui-même, et en particulier par la réalisation de son objet et son but $^{16}$. Par conséquent, pour la CIADH, "l'unique voie» dont l'Etat dispose pour se désengager de sa compétence contentieuse, conformément à la $\mathrm{CADH}$, est « la dénonciation du traité dans son ensemble $»^{17}$.

Dans le cas de la République Dominicaine, on ne se trouve pas stricto sensu devant l'hypothèse d'un retrait de l'acceptation de la compétence contentieuse de la CIADH, mais de la déclaration d'inconstitutionnalité de celle-ci, émise, selon le Tribunal constitutionnel, en violation "manifeste» d'une règle de droit interne d'importance fondamentale. Néanmoins, et malgré la déclaration du Tribunal constitutionnel que la République Dominicaine maintient son engagement envers la Convention américaine des droits de l'homme, la jurisprudence de la Cour interaméricaine des droits de l'homme semblerait indiquer qu'il n'est pas possible qu'un Etat partie à la Convention puisse se désengager de la compétence contentieuse de la juridiction interaméricaine.

En ce qui concerne les organes du système interaméricain, la Commission interaméricaine a condamné la décision du Tribunal constitutionnel et a affirmé qu'elle " n'a aucun fondement en droit international, et ne peut donc avoir d'effet ». Elle a également rappelé qu'au cours des 15 années où l'acceptation de la compétence contentieuse de la 
CIADH par la République Dominicaine avait été en vigueur, celle-ci avait agi dans le respect de l'exercice de cette compétence contentieuse, de sorte que la décision du Tribunal constitutionnel était contraire aux principes de bonne foi et d'estoppel ${ }^{18}$. Pour sa part, en tant que tribunal, la CIADH doit attendre que l'information sur la décision du Tribunal constitutionnel soit officiellement présentée par la Commission interaméricaine, les représentants des victimes ou par l'État lui-même, dans le cadre du contrôle de l'exécution des arrêts contre la République Dominicaine. Après avoir invité les observations des différentes parties, la CIADH devrait normalement se prononcer sur le sujet au travers d'une résolution.

\section{B - Les effets immédiats de la décision du Tribunal constitutionnel : Un impact sur le processus de renforcement du système interaméricain}

19 Le système interaméricain se trouve au centre d'un important processus de rénovation qui vise à apporter des réponses aux défis que confrontent les États membres de l'OEA en matière de droits de l'homme. Ce processus a débuté le 29 juin 2011 lorsque, pendant sa session ordinaire, le Conseil Permanent de l'OEA a décidé de créer un Groupe de Travail pour formuler des recommandations aux Etats membres dans le cadre d'un processus de réflexion sur le fonctionnement de la Commission interaméricaine et de la CIADH par rapport à la CADH, en vue de renforcer le système interaméricain ${ }^{19}$.

En termes généraux, le Groupe de Travail a identifié une série de défis, parmi lesquels ceux liés au but d'atteindre l'universalité du système interaméricain et à assurer la pleine exécution des recommandations et des décisions des organes de ce système. En ce sens, si un État membre de l'OEA n'est pas encore partie à la CADH, il doit réfléchir à la signature et à la ratification, à la ratification ou à l'adhésion à la CADH et à tous les instruments juridiques interaméricains des droits de l'homme, ainsi qu'à l'acceptation de la compétence contentieuse de la CIADH. De cette façon, la conception et la mise en œuvre de stratégies visant à atteindre l'universalité du système interaméricain, en collaboration avec la Commission interaméricaine et la $\mathrm{CIADH}$, les organes politiques compétents de l'OEA, et avec le soutien du Secrétariat général de l'OEA, est considéré comme un objectif parallèle.

21 Comme indiqué par la Cour interaméricaine des droits de l'homme elle-même dans les Lignes directrices 2011-2015 Renforçant la justice interaméricaine, à travers un financement prévisible et harmonieux ${ }^{20}$, document publié dans le cadre du processus de renforcement du système interaméricain, la Cour est le seul tribunal d'Amérique en matière de droits de l'homme, contrairement à tout autre espace institutionnel de l'OEA, qui administre la justice conformément à la CADH et aux autres instruments du système interaméricain. Par conséquent, le travail de la CIADH a un "caractère unique et indélégable » et, en ce sens, celui-ci ne peut être entrepris que par la CIADH ellemême. Ainsi, bien qu'il faille attendre la prise de position de la CIADH sur la décision du Tribunal constitutionnel, il est possible d'affirmer que la seule intention de la République Dominicaine de se désengager de la compétence contentieuse de la CIADH affecte directement le processus de renforcement du système interaméricain en ce qui concerne son universalisation. 
22 En ce sens, la décision du Tribunal constitutionnel a un impact direct sur les différents acteurs du système interaméricain. En effet, outre la Commission interaméricaine, la Coalition des organisations de la société civile en République dominicaine a publié un communiqué de presse appelant tous les citoyens à être vigilants face au « revers juridique et politique » que constitue le désengagement de la compétence contentieuse de la CIADH. ${ }^{21}$ Selon la Coalition, cette situation envoie à la communauté internationale un message négatif sur la République Dominicaine en tant que pays irrespectueux des engagements pris, des droits acquis, et de la dignité de ses citoyens ${ }^{22}$. De même, l'Uruguay, à travers son Ministère des Affaires Étrangères, a déclaré que la décision du Tribunal constitutionnel pourrait avoir des conséquences juridiques susceptibles d'affaiblir l'engagement de la République Dominicaine avec le système interaméricain, «en privant d'efficacité les instruments du système par rapport à ce pays $»^{23}$. Au-delà du système interaméricain, le représentant du Bureau du Haut-Commissariat aux droits de l'homme de l'ONU a fait part de sa préoccupation face à la décision du Tribunal constitutionnel, en espérant que le gouvernement dominicain "reconfirme son engagement" à la $\mathrm{CIADH}$ en notant que «le processus n'a pas encore terminé $»^{24}$.

23 Tant que la décision du Tribunal constitutionnel est maintenue, tout au plus est-il possible d'espérer une ferme décision de la CIADH. Mais de toute évidence, si face à la possible déclaration par la CIADH de l'impossibilité pour la République Dominicaine de se désengager de sa compétence contentieuse à travers la décision du Tribunal constitutionnel, la République Dominicaine suit les pas du Venezuela et dénonce la CADH, le panorama sera encore plus sombre. Ceci est vrai non seulement pour le système interaméricain de façon générale, mais aussi pour les personnes soumises à la juridiction de l'État dominicain, qui seront privées de l'accès subsidiaire à l'organe de contrôle des droits de l'homme par excellence de la région, et qui ne pourront pas invoquer la protection dérivée des droits de l'homme reconnus dans la CADH.

La décision du Tribunal constitutionnel prononçant l'inconstitutionnalité de l'instrument d'acceptation de la compétence contentieuse de la CIADH a suscité une controverse quant à l'existence éventuelle d'un vice du consentement de la République Dominicaine au moment d'accepter cette compétence. Cependant, au-delà des échanges juridiques enrichissants qui ont émergé de la lecture du droit interne dominicain à la lumière de la Convention de Vienne, la décision du Tribunal constitutionnel remet au premier plan la voix des Etats insatisfaits des agissements des organes du système interaméricain et de la force contraignante des arrêts de la CIADH dans l'ordre juridique interne. La décision du Tribunal constitutionnel ne précise pas les conséquences juridiques de la déclaration d'inconstitutionnalité de l'instrument d'acceptation de la compétence contentieuse de la $\mathrm{CIADH}$, mais tout semble indiquer que le principal effet vise à ne pas exécuter les arrêts et résolutions rendus par la CIADH contre la République dominicaine, notamment ceux portant sur la situation des personnes haïtiennes et d'ascendance haïtienne dans le pays. C'est malheureusement une lamentable constatation pour le présent et l'avenir du système interaméricain. 
Tribunal constitutionnel de la République Dominicaine, arrêt TC/0256/14 du 4 novembre 2014.

Les Lettres « Actualités Droits-Libertés » (ADL) du CREDOF (pour s'y abonner) sont accessibles sur le site de la Revue des Droits de l'Homme (RevDH) - Contact

\section{NOTES}

1. La République Dominicaine a proposé Mme. Rhadys Iris Abreu Blondet au poste de magistrat de la CIADH. Elle a occupé cette fonction entre 2006 et 2012. Sur la composition de la CIADH.

2. Loi Organique du Tribunal constitutionnel et des Procédures Constitutionnelles No. 137-11 du 15 juin 2011.

3. CIADH, 8 septembre 2005, Mineures Yean et Bosico c. République Dominicaine, Série C, No. 130. 4. CIADH, 24 octobre 2012, Nadege Dorzema et autres c. République Dominicaine (fond et réparations), Série C, No. 251.

5. CIADH, 28 août 2014, Personnes dominicaines et haïtiennes expulsées c. République Dominicaine (exceptions préliminaires, fond et réparations), Série C, No. 282.

6. Tribunal constitutionnel de la République Dominicaine, arrêt TC/0168/13 du 23 septembre 2013.

7. Loi No. 169-14 du 23 mai 2014.

8. Commission interaméricaine des droits de l'homme, communiqué de presse No. 130/14 du 6 novembre 2014, CIDH condamne la décision du Tribunal constitutionnel de la République Dominicaine.

9. CIADH, 24 septembre 1999, Ivcher Bronstein c. Pérou (compétence), Série C, No. 54.

10. CIADH, 24 septembre 1999, Tribunal constitutionnel c. Pérou (compétence), Série C, No. 55.

11. Ivcher Bronstein §§32-33 ; Tribunal constitutionnel §§31-32.

12. Ivcher Bronstein $\$ 34$; Tribunal constitutionnel $\$ 33$.

13. Ivcher Bronstein $\$ 36$; Tribunal constitutionnel, $\$ 35$.

14. Ivcher Bronstein $\$ 39$; Tribunal constitutionnel 338 .

15. Ivcher Bronstein $\$ 46$; Tribunal constitutionnel $\$ 45$.

16. Ivcher Bronstein $\$ 49$; Tribunal constitutionnel $\$ 48$.

17. Ivcher Bronstein $\S \$ 40,46,50$; Tribunal constitutionnel $\S 39,45,49$.

18. Commission interaméricaine des droits de l'homme, communiqué de presse No. 130/14 du 6 novembre 2014, CIDH condamne la décision du Tribunal constitutionnel de la République Dominicaine.

19. Sur le processus de renforcement du système interaméricain. 
20. CIADH, Lignes directrices 2011-2015 Renforçant la justice interaméricaine, à travers un financement prévisible et harmonieux, 8 juin 2011.

21. Coalition des organisations de la société civile en République Dominicaine, communiqué de presse du 11 novembre 2014.

22. Coalition des organisations de la société civile en République Dominicaine, communiqué de presse du 11 novembre 2014.

23. Ministère des Affaires Étrangères de l'Uruguay, communiqué de presse du 11 novembre 2014, Position de l'Uruguay face à la décision adoptée par le Tribunal constitutionnel de la République Dominicaine.

24. ONU, communiqué de presse du 7 novembre 2014, L'ONU exprime sa préoccupation par rapport à l'arrêt dominicain sur la Cour interaméricaine des droits de l'homme.

\section{RÉSUMÉS}

Le 6 septembre 2012, le Venezuela a présenté un instrument de dénonciation de la Convention américaine des droits de l'homme (CADH) au Secrétaire Général de l'Organisation des Etats Américains (OEA). Conformément à l'article 78 de la $C A D H$, cet instrument est entré en vigueur un an après sa présentation, en septembre 2013. Or, un peu plus d'un an après que la dénonciation par le Venezuela de la CADH soit devenue effective, un autre Etat, la République Dominicaine, a porté un coup dur au processus de renforcement $d u$ système interaméricain des droits de l'homme et à son objectif prioritaire "d'universalisation». En effet, le 4 novembre 2014, le Tribunal constitutionnel de la République Dominicaine a rendu un arrêt qui déclare que l'instrument d'acceptation de la compétence contentieuse de la Cour interaméricaine des droits de l'homme du 19 février 1999, signé par le Président de la République Dominicaine de l'époque, est inconstitutionnel. Et ce, au motif qu'il n'aurait pas été ratifié par le Congrès de la République. La Commission interaméricaine des droits de l'homme, l'Organisation des Nations Unies (ONU), diverses organisations de droits de l'homme de la société civile et même un Etat membre de l'OEA ont condamné cette décision qui interroge quant aux motivations véritables du tribunal constitutionnel. Surtout, une telle solution nationale affaiblit le système interaméricain des droits de l'homme, en faisant écho aux critiques de certains Etats insatisfaits de l'action des organes du système interaméricain et de la force contraignante conférée aux arrêts de la Cour interaméricaine envers les ordres juridiques internes.

\section{AUTEUR}

\section{JUANA MARÍA IBÁÑEZ RIVAS}

Ancienne juriste auprès de la Cour interaméricaine des droits de l'homme, avocate au barreau de Lima (Pérou), membre du Groupe d'Etudes en Droit International et Latino-Américain de la Sorbonne (GEDILAS, rattaché à l'IREDIES) et étudiante du Master 2 Recherche en Droits de l'Homme (Université Paris Ouest) 